\title{
EVALUACIÓN DE TRES LEGUMINOSAS COMO COBERTURAS ASOCIADAS CON MAÍZ EN EL TRÓPICO SUBHÚMEDO ${ }^{1}$
}

\author{
José Bernardino Castillo-Caamal ${ }^{2}$, Jesús Arturo Caamal-Maldonado², Juan José María Jiménez-Osornio², \\ Francisco Bautista-Zúniza ${ }^{3}$, Manuel Jesús Amaya-Castro ${ }^{4}$, Rodrigo Rodríguez-Carrillo ${ }^{4}$
}

\section{RESUMEN}

Evaluación de tres leguminosas como coberturas asociadas con maíz en el trópico subhúmedo. El objetivo fue evaluar el potencial de tres leguminosas como coberturas para mejorar el sistema de producción de maíz. Se evaluaron cuatro tratamientos: 1) maíz solo; 2) maíz y Phaseolus lunatus de ciclo corto de semilla blanca 3) maíz y Phaseolus lunatus de ciclo largo de semilla blanca y 4) maíz y frijol terciopelo (Mucuna sp.) en un diseño experimental de bloques al azar, con cuatro réplicas, durante cuatro ciclos de cultivo (1999-2002) en Yucatán, México. Se midió la biomasa de cultivos, $\mathrm{pH}, \mathrm{N}$ total, mineralización potencial anaerobia del nitrógeno, evolución del $\mathrm{CO}_{2}$, potasio $(\mathrm{K})$, fósforo $(\mathrm{P}) \mathrm{y}$ materia orgánica del suelo, biomasa y frecuencia de arvenses. La cantidad de rastrojo en los tratamientos con leguminosas fue: 3436,3425 y $4018 \mathrm{~kg}$ MS/ha para el primero, segundo y tercer año, respectivamente. El nitrógeno aportado por los rastrojos al maíz fue 25,$8 ; 31,9 ; 52,8$ y $43,4 \mathrm{~kg}$ /ha para los tratamientos 1, 2, 3 y 4, respectivamente. El rendimiento de grano de maíz fue en promedio 841,843 y $460 \mathrm{~kg}$ MS/ha para el primero, segundo y tercer ciclo, respectivamente; sin diferencias entre tratamientos. En el tercer ciclo, el frijol terciopelo disminuyó la biomasa de arvenses de 126 a $58 \mathrm{~g} \mathrm{MS} / \mathrm{m}^{2}$. Las coberturas ejercieron poca influencia en las variables de suelo y rendimiento de grano de maíz.

Palabras clave: Arvenses, nutrimentos, materia

\begin{abstract}
Evaluation of three legumes as cover crops with maize under subhumid tropic. The objective of this work was to evaluate the potential of three legumes as cover crops to improve maize production systems. Four treatments were evaluated: 1) control, maize in monoculture; 2) maize - short-cycle, white seed lima bean (Phaseolus lunatus); 3 ) maize - long cycle, white seed lima bean Phaseolus lunatus), and 4) maize - velvet bean (Mucuna sp.), in a randomized complete blocks design with four replications during four growing cycles (1999-2002), in Yucatán, México. We measured: crop biomass, $\mathrm{pH}$, total $\mathrm{N}$, potential anaerobic mineralization of $\mathrm{N}, \mathrm{CO}_{2}$ evolution, potassium $(\mathrm{K})$, phosphorus $(\mathrm{P})$, and soil organic matter, and biomass and frequency of weeds. Legume stubble residues accumulated on maize crops were: 3436,3425 and $4018 \mathrm{~kg} \mathrm{DM} /$ ha for the first, second and third year, respectively. Nitrogen contributed by stubble material was $25.8,31.9,52.8$, and $43.4 \mathrm{~kg} / \mathrm{ha}$ in treatments 1, 2, 3, and 4, respectively. Maize grain yield was 841, 843 , and $460 \mathrm{~kg} \mathrm{DM} / \mathrm{ha}$ in the first, second and third year, respectively, without significant differences between treatments. During the third year, velvet bean caused a reduction in weed biomass from 126 to $58 \mathrm{~g} \mathrm{DM} / \mathrm{m}^{2}$. Cover crops had little influence on soil variables and maize grain yield.
\end{abstract}

Key words: Weeds, nutrients, organic matter. orgánica.

\footnotetext{
1 Recibido: 29 de julio, 2009. Aceptado: 24 de junio, 2010. La información de este trabajo formó parte de la tesis de Doctorado del primer autor, así como de la tesis de Maestría en Ciencias, del penúltimo y de la tesis de Licenciatura en Biología del último autor.

2 Campus de Ciencias Biológicas y Agropecuarias - Universidad Autónoma de Yucatán. Proyecto: "Evaluación de leguminosas para intensificar la milpa en Yucatán” clave: 980603. jose.castillo@uady.mx; cmaldon@uady.mx; josornio@uady.mx. Carretera 15,5 MéridaXmatkuil, C. P. 97315. AP 4-116 Itzimná, Mérida, Yucatán.

3 Centro de Investigaciones en Geografía Ambiental, Universidad Nacional Autónoma de México (UNAM). leptosol@ciga.unam.mx. Antigua Carretera a Pátzcuaro No. 8701 Col. Ex-Hacienda de San José de La Huerta 58190 Morelia Michoacán, México.

4 Estudiantes de Maestría en Ciencias Manejo y Conservación de Recursos Naturales y Licenciatura en Biología-Universidad Autónoma de Yucatán.mamaya@hotmail.com, biologorodrigo@hotmail.com
} 


\section{INTRODUCCIÓN}

La tradicional práctica del sistema agrícola de roza, tumba y quema (r-t-q), hoy es aplicado por casi 300 millones de campesinos, en un 30\% de los suelos cultivables en el mundo. En México, esta práctica de r$\mathrm{t}$-q se aplica en 5 millones de ha, de las cuales un 10\% se cultivan y las restantes se descansan (Hernández-X et al. 1995).

En la Península de Yucatán, México, se produce maíz, para el consumo de más de un millón de campesinos bajo este sistema (milpa). La baja producción de maíz y su disminución de un año a otro, se atribuye a la competencia de arvenses (Caamal-Maldonado et al. 2001). También se ha considerado que la fertilidad del suelo disminuye de un ciclo a otro como otra causa principal (Weisbach et al. 2002).

La integración de leguminosas de cobertura en los sistemas de producción, es una opción para mejorar la fertilidad del suelo. Estas especies pueden fijar el $\mathrm{N}$ de la atmósfera y mejorar las propiedades físicas y químicas del suelo. También pueden reducir la erosión, aumentar la actividad biológica en el suelo y controlar las arvenses y plagas del suelo, su empleo como alimento o forraje (Binder 1997). Las leguminosas para cobertura deben evaluarse en cuanto a la interferencia (competencia y alelopatía), producción de biomasa. También es importante su papel en el reciclaje de nutrimentos y en la calidad del suelo, entre otras cosas. Además, deben considerarse la distribución estacional de la humedad y su acumulación anual para la siembra de las leguminosas. Ello determina si el manejo de la cobertura será en rotación, intercalación o relevo (Buckles y Barreto 1996).

En el trópico y subtrópico, los frijoles terciopelo (FT) (Mucuna sp.) y espada (Canavalia ensiformis) han tenido mayor difusión. Estas especies de leguminosas presentan rendimiento elevado de biomasa (Kessler 1990, Buckles y Triomphe 1999), mejoran la producción de maíz (Sanginga et al. 1996) y controlan las arvenses (Carsky et al. 1998, Caamal-Maldonado et al. 2001). No obstante, su adopción en el sureste de México ha sido baja debido a que compiten sobre el cultivo principal. Además desplazan otros cultivos tradicionales y poco se conoce de sus uso alimenticio, forraje, etc. (Eilittä y Carsky 2003).

En Yucatán, México, las leguminosas son especies dominantes en la vegetación natural y en la milpa (Terán et al. 1998, Flores 2001). Ellas representan junto con el maíz la base de la alimentación de las comunidades rurales de Yucatán (Terán et al. 1998). Los ibes (Phaseolus lunatus), el espelón (Vigna unguiculata) y el frijol milpero (Phaseolus vulgaris) podrían también utilizarse como coberturas. El objetivo fue evaluar el potencial de tres leguminosas como coberturas para mejorar el sistema de producción de maíz.

\section{MATERIALES Y MÉTODOS}

\section{Ubicación y características del área experimental}

Este trabajo se realizó en la Escuela de Agricultura Ecológica Uyits Ka'an de Maní, Yucatán, México durante cuatro ciclos de cultivo, que abarcaron de 1999 a 2002 . El sitio se localiza a $89^{\circ} 25^{\prime} 11^{\prime \prime}$ longitud oeste y $20^{\circ} 23^{\prime} 17^{\prime \prime}$ latitud norte. El clima es cálido sub-húmedo, Ax (w0) (i) gw". Es el sitio más seco de la región y registra en promedio $1047 \mathrm{~mm} / \mathrm{año}$, con un periodo de medio verano, y la temperatura media anual es de $25,8^{\circ} \mathrm{C}$ (Balam et al. 1999).

El relieve consiste de planicies estructurales con alternancia de lomeríos y depresiones (Lugo y García 1999). Los suelos son del tipo Leptosol réndzico (LPrz) o Lithic Haplustolls y Luvisol ródico (LVro) (Amaya et al. 2005). La vegetación corresponde a selva mediana subcaducifolia. Las especies que predominan con baja actividad humanason: ya'ax-nic (Vitex gaumeri), habín (Piscidia piscipula), pich (Enterolobium ciclocarpum), ya'ax-ché (Ceiba pentandra) (Durán y Olmsted 1999). Sin embargo, en los últimos 20 años hay predominio de vegetación secundaria derivada de las actividades humanas (Durán y Olmsted 1999).

\section{Sitio experimental}

El área experimental consistió de cuatro bloques, seleccionados de acuerdo con su pedregosidad y color del suelo. Las parcelas experimentales fueron de $5 \mathrm{~m} \times 6 \mathrm{~m}$, con área útil de $3 \mathrm{~m} \mathrm{x} 4 \mathrm{~m}$; allí se tomaron muestras de suelo, rendimiento de biomasa de cultivo, de arvenses y grano de maíz.

El experimento se llevó a cabo en la época lluviosa de cada ciclo de cultivo. Se utilizó el maíz de la variedad Nal-Xoy, de grano amarillo (Dzib y Paczka 2002). Las siembras del maíz fueron en las fechas: en 
1999, el 12 de junio; en 2000, el 9 de junio; en 2001, el 13 de junio, y en 2002, el 6 de junio.

La distancia de siembra fue de un metro entre hileras y posturas; una densidad de 40000 semillas/ ha. Las leguminosas fueron intercaladas entre $25 \mathrm{y}$ 35 días después de la siembra (DDS) del maíz; ello estuvo relacionado con la disponibilidad de humedad. Se sembraron a $1,0 \mathrm{~m}$ entre hileras y $0,50 \mathrm{~m}$ dentro de hileras, con dos semillas por postura. Se hicieron dos deshierbes: entre los 25 y 35 DDS y entre los 70 y 75 DDS. El maíz fue cosechado después del secado de la mazorca, antes que las leguminosas alcanzaron su máxima cobertura.

\section{Variables de respuesta}

Antes de efectuar la siembra se tomaron muestras compuestas de suelo, a una profundidad de 0 a $10 \mathrm{~cm}$; éstas se secaron en sombra y se tamizaron con una criba de 0,417 mm de diámetro. En mayo de 2000, mayo de 2001 y mayo de 2002 se obtuvieron nuevas muestras de suelos.

El material edáfico se sometió a los siguientes análisis: pH en agua relación 1:2,5 usando el método potenciométrico (Houba et al. 1988), carbono orgánico por el método Walkley y Black (Nelson y Sommers 1982, Okalebo et al. 1993), nitrógeno total por el método Kjeldhal (Anderson e Ingram 1993), potasio intercambiable extraído con acetato de amonio (Okalebo et al. 1993), fósforo Olsen (Anderson e Ingram 1993), evolución de $\mathrm{CO}_{2}$ (Anderson e Ingram 1993 y Parkin et al. 1996) y mineralización potencial anaerobia del nitrógeno (MPAN) con incubación de siete días (Houba et al. 1988 y Drinkwater et al. 1996).

Se midió el rendimiento de grano de maíz y de leguminosas, rastrojo de ambos, y la biomasa total. Después de cada ciclo de cultivo se tomaron muestras de hojarasca, usando marcos de $50 \mathrm{~cm}$ x $50 \mathrm{~cm}$.

En el cuarto ciclo, no se tomaron las muestras de cultivos por la inundación causada por el huracán Isidoro; una vez que se secó el área fueron tomadas las muestras de suelo. Se determinaron los contenidos de nitrógeno total y fósforo en la biomasa de maíz, leguminosas y hojarasca del ciclo 2000-2001. Se estimaron los balances de nitrógeno y fósforo a través de las diferencias entre entradas-salidas de cada variable; las entradas estuvieron representadas por: rastrojo de maíz, rastrojo de leguminosas y hojarasca; las salidas, por: grano de maíz, olote de maíz y grano de leguminosas.

Se estimó la frecuencia relativa y la biomasa de arvenses por medio de marcos de $50 \mathrm{~cm}$ x $50 \mathrm{~cm}$. En el primer ciclo se tomaron muestras en tres fechas; en los subsecuentes, excepto en el cuarto, se realizaron dos muestreos, tomando cinco muestras en la unidad experimental.

\section{Diseño experimental}

Se utilizó un diseño experimental de bloques completos al azar con cuatro réplicas y cuatro tratamientos. Los tratamientos fueron: 1) control, maíz solo (MSOL); 2) maíz intercalado con frijol lima de ciclo corto de grano blanco (Phaseolus lunatus), (MICB); 3) maíz intercalado con frijol lima de ciclo largo de grano blanco (Phaseolus lunatus) (MIGB) y 4) maíz intercalado con frijol terciopelo de grano cenizo (Mucuna $s p$ ) (MUNA). Los datos fueron sometidos a los análisis de varianza de acuerdo con el diseño experimental empleado (Steel y Torrie 1988).

Los datos de biomasa de arvenses fueron transformados a logaritmo base 10. Para el rendimiento de grano y rastrojo del maíz se utilizó la covariable número de plantas para el análisis estadístico. Para las variables del suelo los valores iniciales de cada una fueron utilizados como covariables. Se hizo la comparación de medias de tratamientos por la prueba de rango múltiple de Duncan. Por otra parte, las variables del suelo fueron sometidas a un análisis de regresión lineal.

\section{RESULTADOS Y DISCUSIÓN}

Las precipitaciones anuales fueron de 1359, 1106 y $1272 \mathrm{~mm}$ para 1999, 2000 y 2001, respectivamente. Las lluvias de mayo a octubre, fueron de 1161, 898 y $1001 \mathrm{~mm}$ para 1999, 2000 y 2001, respectivamente, de acuerdo con las hojas estadísticas proporcionadas por la delegación en Yucatán de la Comisión Nacional del Agua. Con estas precipitaciones, el rendimiento de maíz fue 841 y $843 \mathrm{~kg} / \mathrm{ha}$ para el primero y segundo año, respectivamente, en el tercer año, el rendimiento se redujo a $526 \mathrm{~kg} / \mathrm{ha}$. Estas medias se ubican en el límite inferior reportado para la variedad Nal-Xoy en Yucatán (Dzib y Ortega-Paczka 2002). 
En ninguno de los tres años se encontró efecto de las leguminosas sobre el rendimiento de la gramínea (Cuadro 1). Resultados similares fueron encontrados en el noreste de Belice, después de tres ciclos de cultivo de frijol terciopelo asociado al maíz (Coultas et al. 1996). En el estado mexicano de Chiapas, el frijol terciopelo tampoco mejoró el rendimiento de maíz (Pool et al. 1997). Ayala y Basulto (2004) tampoco encontraron efecto del frijol terciopelo en el rendimiento del grano de maíz en Yucatán. En condiciones de temporal, los frijoles terciopelo y Canavalia, disminuyeron el rendimiento de grano de maíz, al competir con él (Bernabé 1998).

Una contribución de las leguminosas en sistemas de cultivos asociados, es la mejora de los nutrimentos en particular del nitrógeno. Stern (1993) indica que este elemento puede ser transferible en forma directa a la planta no leguminosa; de igual modo es transferido al cultivo por la descomposición de la biomasa en ciclos subsecuentes. Ello al parecer no ocurrió en este experimento por los bajos rendimientos del grano de maíz obtenidos.

La biomasa registrada en las tres leguminosas, mayor de $2000 \mathrm{~kg} / \mathrm{ha}$, es suficiente para cumplir la función de cobertura, al aportar material orgánico al suelo (Lobo-Burle et al. 1992). La ausencia de efecto sobre el rendimiento del grano, se atribuye a la asincronía de la producción de la biomasa; ello reduce su aprovechamiento por la gramínea (Amaya et al. 2005). De hecho, el impacto de las coberturas está asociado con la descomposición de su biomasa en sistemas de rotación (Sanginga et al. 1996, Buckles y Triomphe 1999, Eilittä et al. 2003a).

Otros sistemas de manejo aprovechan la humedad disponible previa al cultivo principal; así las coberturas producen biomasa en una parte del año (Lathwel 1990, Lobo-Burle et al. 1992, Carsky et al. 1998, Wortmann et al. 2000). En Yucatán por la baja precipitación y suelos permeables, el manejo de coberturas, se restringe al intercalado o relevo; ello permite aprovechar la humedad estacional y evitar el desplazamiento del cultivo principal. Entonces, las coberturas deben crecer con el cultivo, producir biomasa y no comprometer el rendimiento del cereal.

\section{Acumulación de biomasa}

En el ciclo 1999-2000 la biomasa total fue similar entre tratamientos $(\mathrm{P}>0,05)$. En los ciclos 2000-2001 y
2001-2002, destacaron el maíz asociado al frijol lima de ciclo largo y frijol terciopelo (Cuadro 1). No obstante, la biomasa correspondiente al rastrojo, presentó diferencias en el primero y tercer ciclo. En el primer ciclo, la inclusión de las leguminosas contribuyó con una mayor acumulación de biomasa a partir del rastrojo. En el tercer ciclo los menores rendimientos correspondieron al frijol lima de ciclo corto y al maíz en monocultivo.

Se presentó una baja concentración de $\mathrm{N}$ de la hojarasca menor que a lo encontrado por Buckles y Triomphe (1999). Además, se requiere considerar la descomposición del material orgánico y la mineralización de $\mathrm{N}$; en el frijol terciopelo estos procesos son rápidos, pues el $\mathrm{N}$ es utilizado en un plazo corto, mientras el maíz crece (Buckles y Triomphe 1999). Este fenómeno ocurre también en las otras leguminosas por sus concentraciones altas de nitrógeno y una baja relación C/N (Jensen y Castellanos 1994, Armendáriz 1998). Aunque la leguminosa produce suficiente biomasa, la producción, descomposición y aprovechamiento, estuvo en asincronía al cultivo del maíz; en efecto, el mayor potencial de la mineralización anaerobia del nitrógeno del suelo ocurrió en marzo (Amaya et al. 2005).

\section{Biomasa y frecuencia relativa de arvenses}

Las coberturas, por lo general, disminuyen la biomasa de las arvenses durante el ciclo de cultivo. Sin embargo, en este estudio las leguminosas no redujeron $(\mathrm{P}>0,05)$ el desarrollo de las arvenses en los dos primeros ciclos de cultivo. Su impacto se manifestó en la segunda fecha de muestreo del tercer ciclo, cuando contribuyeron al disminuir la biomasa de esas plantes (Cuadro 2).

No hubo cambios sustanciales en la biomasa de arvenses en los diferentes tratamientos con respecto al testigo, lo que se relacionó con el tiempo de cultivo (Caamal-Maldonado et al. 2001). En el primer ciclo de cultivo, el área dispone del mismo banco de semillas y las arvenses que germinan en esta etapa inicial no generan una interferencia mayor. Por el contrario, después de dos o tres ciclos de cultivo la abundancia de las arvenses fue mayor (Caamal-Maldonado et al. 2001). Así, las coberturas pueden evitar la germinación de nuevos individuos o provocar que los que alcancen a germinar no se desarrollen hasta reproducirse.

En particular, frijol terciopelo redujo la biomasa de las arvenses, lo que coincide con otros trabajos en la región (Caamal-Maldonado et al. 2001, Ayala 
Cuadro 1. Producción de biomasa (kg MS/ha) en el sistema de producción de maíz intercalado con tres leguminosas de cobertura, durante tres ciclos de cultivo (1999 a 2002). Maní, Yucatán, México.

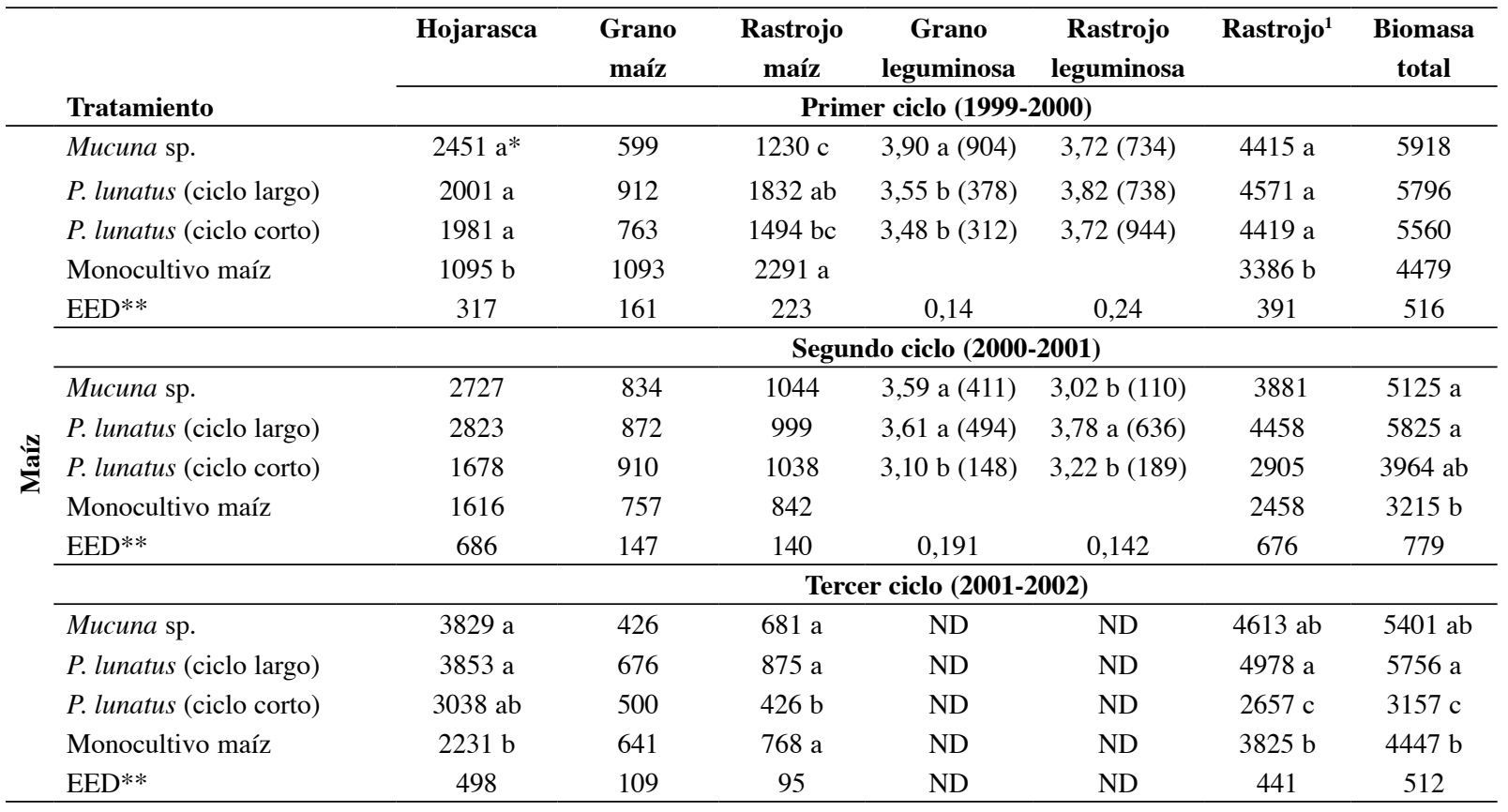

* Literales diferentes dentro de las columnas indican diferencias significativas $(0,05)$.

** EED: Error estándar de la diferencia entre dos medias.

${ }^{1}$ Incluye rastrojo de maíz, rastrojo de leguminosas y hojarasca; cifras entre paréntesis corresponde a los valores sin la transformación.

ND: no hay datos de grano y rastrojo de leguminosas, por exceso de humedad.

Cuadro 2. Efecto la asociación de leguminosas de cobertura con el maíz sobre la biomasa ( $\left.\mathrm{g} \mathrm{MS} / \mathrm{m}^{2}\right)$ de arvenses, durante tres ciclos de cultivo (1999-2001), en Maní, Yucatán, México.

\begin{tabular}{|c|c|c|c|c|c|c|c|c|}
\hline & \multirow{3}{*}{ Tratamientos } & \multicolumn{3}{|c|}{ Ciclo 1} & \multicolumn{2}{|c|}{ Ciclo 2} & \multicolumn{2}{|c|}{ Ciclo 3} \\
\hline & & \multicolumn{7}{|c|}{ Fechas de muestreo } \\
\hline & & Jul 1999 & Ago 1999 & Sep 1999 & Ago 2000 & Oct 2000 & Ago 2001 & Oct 2001 \\
\hline \multirow{5}{*}{ 莺 } & Mucuna sp. & $1,80(75)^{1}$ & $1,12(12)$ & $1,10(12)$ & $1,81(70)$ & $1,76(61)$ & $1,69(54)$ & $1,76 b^{2}(58)$ \\
\hline & P. lunatus (ciclo largo) & $1,50(34)$ & $1,37(25)$ & $1,37(26)$ & $1,91(83)$ & $1,84(71)$ & $1,82(69)$ & 2,07 a (123) \\
\hline & P. lunatus (ciclo corto) & $1,52(32)$ & $1,36(24)$ & $1,27(19)$ & $1,71(52)$ & $1,71(52)$ & $1,86(75)$ & 1,98 a (98) \\
\hline & Monocultivo maíz & $1,57(52)$ & $1,11(12)$ & $1,35(25)$ & $1,88(82)$ & $2,02(109)$ & $1,86(76)$ & $2,07 \mathrm{a}(126)$ \\
\hline & $\mathrm{EED}^{3}$ & 0,161 & 0,124 & 0,137 & 0,099 & 0,106 & 0,091 & 0,081 \\
\hline
\end{tabular}

${ }^{1}$ Cifras dentro de paréntesis corresponden a las medias originales, sin transformación logarítmica.

${ }^{2}$ Literales diferentes dentro de columnas indican diferencias significativas $(0,05)$.

${ }^{3}$ EED: Error estándar de la diferencia entre dos medias.

y Basulto 2004). La reducción de la biomasa de las arvenses se atribuye a la etiolación causada por la sombra del frijol terciopelo. Además, se han señalado que el frijol terciopelo posee compuestos alelopáticos, útiles en el manejo de arvenses (Fujii et al. 1992, Caamal-Maldonado et al. 2001). 


\section{Arvenses}

En las arvenses la frecuencia relativa mayor correspondió a xtaulmii (Ageratum gaumeri) y tsaitsá (Neomillspaugia emarginata), especies que dejan reservorios reproductivos subterráneos (Cuadro 3); la primera es una herbácea, mientras que la segunda es un arbusto. En el tratamiento con frijol terciopelo aunque su frecuencia relativa es elevada, la biomasa fue reducida, lo que facilita su control.

Las especies de arvenses con mayor frecuencia y que contribuyen con más biomasa se reproducen vegetativamente, y tienen reservorios que contribuyen a que los nuevos propágulos puedan establecerse. Los efectos de la interferencia (competencia y alelopatia) de las coberturas podrían mostrarse después de varios años de manejo (Cuadro 3).

\section{Concentración de nitrógeno y de fósforo en la plan- ta, acumulación y flujo en el sistema}

La concentración del nitrógeno registrado en la biomasa de maíz coincide con otras variedades de este género (Reyes 1990, Romero 2000). En las leguminosas las concentraciones de este elemento concuerdan a lo reportado por NAS (1979) y Ayala-Burgos et al. (2003). La acumulación del nitrógeno con las coberturas fue superior que en el testigo (Cuadro 4).

La cantidad de $\mathrm{N}$ apenas alcanzó la cantidad mínima indicada por Lobo-Burle et al. (1992); en maíz y semilla grande blanco: $53 \mathrm{~kg} \mathrm{~N} / \mathrm{ha}$; en maíz y frijol terciopelo: $43 \mathrm{~kg} \mathrm{~N} / \mathrm{ha}$ (Cuadro 5). Así, utilizar todo el grano procedente de las coberturas para alimento o forraje, reduce la cantidad de nitrógeno del sistema (Jensen y Castellanos 1994, van Kessel y Hartley

Cuadro 3. Efecto de leguminosas de cobertura asociadas con maíz sobre la frecuencia relativa (\%) de arvenses, durante el ciclo 2002-2001, en Maní, Yucatán, Méxic.

\begin{tabular}{lcccc}
\hline & \multicolumn{2}{c}{ Maíz } \\
\cline { 2 - 5 } Especies de arvenses & $\begin{array}{c}\text { Maíz en } \\
\text { monocultivo }\end{array}$ & $\begin{array}{c}\text { P. lunatus } \\
\text { (ciclo largo) }\end{array}$ & Mucuna sp. & $\begin{array}{c}\text { P. lunatus } \\
\text { (ciclo corto) }\end{array}$ \\
\cline { 2 - 5 } & \multicolumn{4}{c}{ Frecuencia relativa (\%) } \\
\hline Xtaulmii (Ageratum gaumeri) & 15,96 & 15,19 & 14,46 & 20,20 \\
Sac-xiu (Walteria americana) & 7,45 & 1,27 & 1,20 & 0,0 \\
Xkansuuk (Digitaria ciliaris) & 4,26 & 12,26 & 6,02 & 10,10 \\
Nichii-yuc (Corchorus siliquosus) & 4,26 & 10,3 & 7,23 & 6,06 \\
Xtus-iik-xiu (no identificada) & 5,32 & 6,33 & 8,43 & 6,06 \\
Xtokaabán (Eupatorium adoratum) & 1,06 & 2,53 & 8,43 & 3,03 \\
Xtsaitsá (Neomillspaugia emarginata) & 19,15 & 18,99 & 16,87 & 18,80 \\
\hline
\end{tabular}

Cuadro 4. Concentración de nitrógeno (\%) en los componentes de maíz y de leguminosas asociadas como cultivos de cobertura, en el ciclo 2000-2001. Maní, Yucatán, México.

\begin{tabular}{lccccc}
\hline \multirow{2}{*}{ Componente de la planta } & \multicolumn{5}{c}{ Maíz } \\
\cline { 2 - 5 } & $\begin{array}{c}\text { Maíz en } \\
\text { monocultivo }\end{array}$ & $\begin{array}{c}\text { P. lunatus } \\
\text { (ciclo corto) }\end{array}$ & $\begin{array}{c}\text { P. lunatus } \\
\text { (ciclo largo) }\end{array}$ & Mucuna sp. & EED \\
\cline { 2 - 6 } & \multicolumn{7}{c}{$\mathbf{N}$ (\%) } \\
\hline Grano de maíz & 1,35 & 1,54 & 1,49 & 1,46 & 0,184 \\
Olote de maíz & 0,55 & 0,47 & 0,55 & 0,53 & 0,053 \\
Rastrojo de maíz & 0,54 & 0,70 & 0,67 & 0,65 & 0,052 \\
Hojarasca & 1,32 & 1,28 & 1,39 & 1,38 & 0,102 \\
Grano leguminosa & - & 3,65 & 3,12 & 3,34 & 0,207 \\
Rastrojo leguminosa & - & $1,36 \mathrm{a}^{2}$ & $1,20 \mathrm{a}$ & $0,65 \mathrm{~b}$ & 0,078 \\
\hline
\end{tabular}

${ }^{1}$ Error estándar para comparar MIGB con MICB, MSOL o MUNA.

${ }^{2}$ Literales diferentes dentro de hileras indican diferencias significativas $(0,05)$. EED: Error estándar de la diferencia entre dos medias. 
Cuadro 5. Acumulación de nitrógeno ( $\mathrm{kg} / \mathrm{ha}$ ) en los componentes de la asociación maíz y leguminosas como coberturas, en el ciclo 2000-2001, en Maní, Yucatán, México.

\begin{tabular}{|c|c|c|c|c|c|}
\hline \multirow{3}{*}{$\begin{array}{l}\text { Componente de la } \\
\text { planta }\end{array}$} & \multicolumn{4}{|c|}{ Maíz } & \multirow[b]{2}{*}{ EED $^{1}$} \\
\hline & $\begin{array}{c}\text { Maíz en } \\
\text { monocultivo }\end{array}$ & $\begin{array}{l}\text { P. lunatus } \\
\text { (ciclo corto) }\end{array}$ & $\begin{array}{l}\text { P. lunatus } \\
\text { (ciclo largo) }\end{array}$ & Mucuna sp. & \\
\hline & \multicolumn{4}{|c|}{ N (kg/ha) } & \\
\hline Grano de maíz & 9,8 & 14,0 & 12,5 & 11,5 & 3,04 \\
\hline Olote de maíz ${ }^{1}$ & 0,68 & 0,79 & 0,80 & 0,85 & 0,16 \\
\hline Rastrojo de maíz & 4,6 & 7,3 & 6,58 & 6,85 & 0,96 \\
\hline Hojarasca & 20,5 & 21,8 & 38,8 & 38,0 & 10,7 \\
\hline Grano leguminosa & & 5,6 & 15,1 & 14,0 & 5,1 \\
\hline Rastrojo leguminosa & & $2,57 \mathrm{~b}$ & $7,68 \mathrm{a}$ & $0,71 \mathrm{~b}$ & 1,4 \\
\hline Total N/sistema & $36,2 \mathrm{~b}$ & $52,2 \mathrm{a}$ & $81,2 \mathrm{a}$ & $71,8 \mathrm{a}$ & 13,6 \\
\hline Entradas - salidas & 25,8 & 31,9 & 52,9 & 43,4 & \\
\hline
\end{tabular}

Entrada $=$ rastrojo de maíz, rastrojo de leguminosas y hojarasca. Salidas = grano de maíz, olote de maíz y grano de leguminosas. EED=Error estándar de la diferencia entre dos medias. ${ }^{1}$ : Error estándar de la diferencia entre dos medias con diferente número de repeticiones.

Literales diferentes entre las hileras indican diferencias significativas.

2000). Entonces, podrían seleccionarse coberturas con bajos índices de cosecha, pero ello comprometería los productos para autoconsumo o venta (McDonagh y Hillyer 2003).

La concentración de $\mathrm{P}$ en el rastrojo del maíz asociado con $P$. lunatus ciclo largo y frijol terciopelo (Cuadro 6) concuerda con Ile et al. (1996); ellos sembraron maíz en áreas precedidas del cultivo de frijol terciopelo en suelos ácidos. Pero, difiere de lo obtenido obtenido por Eilittä et al. (2003b) al encontrar mayor P en la hoja de la mazorca con frijol terciopelo.

La mayor concentración de $\mathrm{P}$ en el rastrojo del maíz puede deberse a su mayor disponibilidad, explicado por la disminución del pH del suelo causada por el frijol lima de ciclo largo y el frijol terciopelo. En suelos alcalinos la absorción del fósforo por las plantas, es inhibido por complejos de apatita (Benzing 2001). La mayor concentración de $\mathrm{P}$ en el rastrojo de

Cuadro 6. Concentración de fósforo (mg P/kg MS) en componentes de las plantas de maíz y leguminosas de cobertura, en el ciclo 2000-2001, en Maní, Yucatán, México.

\begin{tabular}{|c|c|c|c|c|c|}
\hline \multirow[b]{2}{*}{ Componente } & \multicolumn{4}{|c|}{ Maíz } & \multirow[b]{2}{*}{ EED $^{1}$} \\
\hline & $\begin{array}{c}\text { Maíz en } \\
\text { monocultivo }\end{array}$ & $\begin{array}{c}P . \text { lunatus } \\
\text { (ciclo corto) }\end{array}$ & $\begin{array}{c}\text { P. lunatus } \\
\text { (ciclo largo) }\end{array}$ & Mucuna sp. & \\
\hline Grano de maíz ${ }^{1}$ & 906 & 1361 & 877 & 990 & 319 \\
\hline Olote de maíz ${ }^{1}$ & 578 & 530 & 458 & 554 & 234 \\
\hline Rastrojo de maíz ${ }^{1}$ & $796 \mathrm{~b}$ & $805 b^{*}$ & $1166 a b$ & $1468 \mathrm{a}$ & 193 \\
\hline Hojarasca $^{2}$ & 1407 & 1599 & 1228 & 1303 & 204 \\
\hline Grano de leguminosa ${ }^{2}$ & & $798 \mathrm{~b}$ & $610 \mathrm{~b}$ & $1274 \mathrm{a}$ & 184 \\
\hline Rastrojo leguminosa ${ }^{2}$ & & $685 \mathrm{a}$ & $658 \mathrm{a}$ & $473 \mathrm{~b}$ & 62 \\
\hline
\end{tabular}

* Letras diferentes dentro de líneas indican diferencias significativas $(0,05)$.

EED: error estándar de la diferencia entre dos medias.

${ }^{1}$ : Error estándar de la diferencia entre dos medias con diferente número de repeticiones.

${ }^{2}$ : Error estándar de la diferencia entre dos medias con igual número de repeticiones. 
las semillas de $P$. lunatus se debió a que al momento del muestreo gran parte del material estaba verde.

Contrario a lo reportado por Buckles y Triomphe (1999), las leguminosas aportaron poco $\mathrm{P}$ total (Cuadro 7) . La proporción del P en la hojarasca del sistema de maíz - mucuna fue elevado; la diferencia es por una mayor cantidad de biomasa que registraron respecto a este experimento.

\section{Propiedades del suelo}

En ninguno de los cuatro ciclos se registró efecto de las leguminosas en las propiedades del suelo; sólo se registró el descenso del pH (Cuadro 8). Este hecho se corresponde con la ausencia de respuesta sobre los rendimientos de maíz. La misma situación fue reportada en el frijol terciopelo por Coutlas et al. (1996) y por Quiroga-Madrigal (2000), en Canavalia ensiformis. Dichos autores no encontraron efectos sobre la mineralización potencial del nitrógeno del suelo y en $\mathrm{P}$ y K ; ello, durante tres ciclos de cultivo continuo en suelos luvisoles, Por el contrario Buckles y Triomphe (1999) registraron incrementos en $\mathrm{P}$ en la rotación de maíz y frijol terciopelo en laderas de Honduras.

La acidificación de los suelos por el manejo de las coberturas difiere de otras experiencias (Quiroga-Madrigal 2000, Buckles y Triomphe 1999). Sin embargo, en suelos calcáreos la acidificación de éstos, puede favorecer la asimilación del fósforo contenido en complejos de apatita (Benzing 2001).

La ausencia de cambios en los contenidos de materia orgánica del suelo concuerda con lo reportado por Quiroga-Madrigal (2000); él trabajó con frijol espada y maíz durante tres años; Coultas et al. (1996) con maíz y frijol terciopelo durante tres años en Gleysoles; asimismo con Bernabé (1998) luego de cuatro ciclos agrícolas. No obstante, en un ambiente de mayor precipitación pluvial, se incrementó la MO del suelo con frijol terciopelo. Este registro se llevó a cabo durante un periodo de 14 años en rotación (Buckles y Triomphe 1999). La ausencia de efectos de las coberturas sobre las propiedades del suelo coincide con trabajos en condiciones de precipitación similar; también en esos casos los periodos de cultivos fueron de tres a cuatro años (Coultas et al. 1996, Martínez y Morales 1997, Bernabé 1998, Quiroga-Madrigal 2000). En esas condiciones, se restringe la acumulación de biomasa misma que se encuentra desfasada con su utilización por el cultivo.

\section{Tendencias de las propiedades del suelo durante los ciclos de cultivo}

Hubo cambios en la respiración del suelo $(\mathrm{P}<0,001)$, MPAN $(\mathrm{P}<0,001)$, en los contenidos de fósforo $(\mathrm{P}<0,001)$, potasio $(\mathrm{P}<0,05)$ y materia orgánica

Cuadro 7. Acumulación de fósforo en los componentes de plantas de maíz y leguminosas en el ciclo de cultivo (2000-2001).

\begin{tabular}{|c|c|c|c|c|c|}
\hline \multirow{3}{*}{$\begin{array}{l}\text { Componente de } \\
\text { la planta }\end{array}$} & \multicolumn{4}{|c|}{ Maíz } & \multirow{3}{*}{ EED ${ }^{1}$} \\
\hline & $\begin{array}{c}\text { Maíz en } \\
\text { monocultivo }\end{array}$ & $\begin{array}{c}\text { P. lunatus } \\
\text { (ciclo corto) }\end{array}$ & $\begin{array}{c}\text { P. lunatus } \\
\text { (ciclo largo) }\end{array}$ & Mucuna sp. & \\
\hline & \multicolumn{4}{|c|}{$\mathbf{N}(\%)$} & \\
\hline Grano de maíz & 0,66 & 1,1 & 0,71 & 0,7 & 0,22 \\
\hline Olote de maíz1 & 0,07 & 0,09 & 0,07 & 0,07 & 0,036 \\
\hline Rastrojo de maíz & $0,47 \mathrm{c}$ & $0,70 b c^{2}$ & $1,17 \mathrm{ab}$ & $1,59 \mathrm{a}$ & 0,27 \\
\hline Hojarasca & 2,32 & 2,26 & 3,46 & 3,46 & 0,85 \\
\hline Grano leguminosa & & 0,13 & 0,39 & 0,51 & 0,22 \\
\hline Rastrojo leguminosa & & $0,14 \mathrm{~b}$ & 0,4 & 0,045 & 0,06 \\
\hline Total P/sistema & $3,55 \mathrm{~b}$ & $4,72 \mathrm{ab}$ & $6,17 \mathrm{a}$ & 6,4 & 0,98 \\
\hline Entradas - salidas & 2,82 & 3,4 & 4,99 & 5,12 & \\
\hline
\end{tabular}

${ }^{1}$ EED: Error estándar de la diferencia entre dos medias.

${ }^{2}$ Literales diferentes dentro de hileras indican diferencias significativas $(0,05)$. 
$(\mathrm{P}<0,001)$; no los hubieron en los contenidos del nitrógeno total del suelo $(\mathrm{P}>0,05)$ (Figura 1).

La materia orgánica disminuyó, mientras que la mineralización potencial anaerobia del nitrógeno se incrementó con el tiempo (Cuadro 8). La reducción de estas variables del suelo (Figura 1) concuerda con trabajos previos realizados en regiones tropicales subhúmedas. Dicha tendencia es limitante para el

Cuadro 8. Propiedades del suelo en el cultivo de maíz asociado con leguminosas de cobertura en ciclos de producción consecutivos (1999- 2002), en Maní, Yucatán, México.

\begin{tabular}{|c|c|c|c|c|c|}
\hline \multirow{3}{*}{ Variables } & \multicolumn{5}{|c|}{ Maíz } \\
\hline & $\begin{array}{c}\text { Maíz en } \\
\text { monocultivo }\end{array}$ & $\begin{array}{l}\text { P. lunatus } \\
\text { (ciclo corto) }\end{array}$ & $\begin{array}{l}\text { P. lunatus } \\
\text { (ciclo largo) }\end{array}$ & Mucuna sp. & EED $^{1}$ \\
\hline & \multicolumn{5}{|c|}{ Ciclo 1999-2000 (previo al primer ciclo de cultivo) } \\
\hline $\mathrm{pH}$ & 8,19 & 8,02 & 7,97 & 8,13 & \\
\hline $\mathrm{N}$ total $\mathrm{g} / \mathrm{kg}$ & 4,17 & 4,28 & 4,26 & 4,36 & \\
\hline Materia orgánica g/100 & 13,24 & 13,10 & 13,19 & 12,55 & \\
\hline Fósforo $\mathrm{mg} / \mathrm{kg}$ Materia seca & 347,6 & 215,4 & 238,6 & 229,0 & \\
\hline Potasio mg/kg Materia seca & 1445 & 1304 & 1638 & 1077 & \\
\hline $\mathrm{CO}_{2} \mathrm{mg} / \mathrm{kg}$ Materia seca & 891,4 & 828,1 & 911,6 & 890,2 & \\
\hline \multirow{2}{*}{$\begin{array}{l}\text { Mineralización potencial anaerobia } \\
\text { nitrógeno } \mathrm{mg}\left(\mathrm{NH}_{4}\right) / \mathrm{kg} \text { Materia seca }\end{array}$} & 25,32 & 28,32 & 10,50 & 27,02 & \\
\hline & \multicolumn{5}{|c|}{ Ciclo 2000-2001 } \\
\hline $\mathrm{pH}$ & $8,10 \mathrm{a}$ & $7,91 \mathrm{c}$ & $7,83 \mathrm{bc}$ & $7,96 \mathrm{abc}$ & 0,070 \\
\hline $\mathrm{N}$ total $\mathrm{g} / \mathrm{kg}$ & 4,57 & 4,48 & 4,82 & 3,95 & 0,52 \\
\hline Materia orgánica g/100 & 13,6 & 12,0 & 13,1 & 9,7 & 1,59 \\
\hline Fósforo $\mathrm{mg} / \mathrm{kg}$ Materia seca & 166 & 198 & 195 & 178 & 17,73 \\
\hline Potasio mg/kg Materia seca & 1268 & 1303 & 1462 & 1304 & 134,1 \\
\hline $\mathrm{CO}_{2} \mathrm{mg} / \mathrm{kg}$ Materia seca & 532 & 626 & 628 & 617 & 94,6 \\
\hline \multirow{3}{*}{$\begin{array}{l}\text { Mineralización potencial anaerobia del } \\
\text { nitrógeno } \mathrm{mg}\left(\mathrm{NH}_{4}\right) / \mathrm{kg} \text { Materia seca }\end{array}$} & & & & & \\
\hline & 15,6 & 15,4 & 14,8 & 16,1 & 1,23 \\
\hline & \multicolumn{5}{|c|}{ Ciclo 2001-2002 } \\
\hline $\mathrm{pH}$ & $7,97 \mathrm{a}$ & $7,63 \mathrm{c}$ & $7,71 \mathrm{bc}$ & $7,84 \mathrm{ab}$ & 0,09 \\
\hline $\mathrm{N}$ total $\mathrm{g} / \mathrm{kg}$ & 4,54 & 4,39 & 4,65 & 4,04 & 0,54 \\
\hline Materia orgánica (\%) & 10,9 & 10,2 & 10,6 & 9,4 & 1,47 \\
\hline Fósforo $\mathrm{mg} / \mathrm{kg}$ Materia seca & 224 & 210 & 226 & 220 & 15,9 \\
\hline Potasio mg/kg Materia seca & 1143 & 985 & 1426 & 1139 & 172 \\
\hline $\mathrm{CO}_{2} \mathrm{mg} / \mathrm{kg}$ Materia seca & 982 & 1013 & 1027 & 999 & 85,6 \\
\hline \multirow{3}{*}{$\begin{array}{l}\text { Mineralización potencial anaerobia } \\
\text { nitrógenomg }\left(\mathrm{NH}_{4}\right) / \mathrm{kg} \text { Materia seca }\end{array}$} & 23,18 & 24,64 & 25,95 & 26,65 & 1,74 \\
\hline & & & & & \\
\hline & \multicolumn{5}{|c|}{ Ciclo 2002-2003 } \\
\hline $\mathrm{pH}$ & $7,85 \mathrm{a}$ & $7,29 \mathrm{c}$ & $7,32 \mathrm{c}$ & $7,52 \mathrm{~b}$ & 0,078 \\
\hline $\mathrm{N}$ total $\mathrm{g} / \mathrm{kg}$ & 4,48 & 4,40 & 4,58 & 4,00 & 0,33 \\
\hline Materia orgánica (\%) & 10,37 & 9,85 & 11,04 & 9,54 & 1,30 \\
\hline Fósforo $\mathrm{mg} / \mathrm{kg}$ Materia seca & 143 & 116 & 126 & 130 & 16,10 \\
\hline Potasio $\mathrm{mg} / \mathrm{kg}$ Materia seca & 1123 & 1074 & 1182 & 1032 & 61,5 \\
\hline $\mathrm{CO}_{2} \mathrm{mg} / \mathrm{kg}$ Materia seca & 598 & 667 & 786 & 671 & 137,5 \\
\hline $\begin{array}{l}\text { Mineralización potencial anaerobia del } \\
\text { nitrógeno } \mathrm{mg}\left(\mathrm{NH}_{4}\right) / \mathrm{kg} \text { Materia seca }\end{array}$ & 50,2 & 57,7 & 64,4 & 55,1 & 6,7 \\
\hline
\end{tabular}

Letras diferentes dentro de hileras indican diferencias estadísticas $(0,05$ y 0,001).

${ }^{1}$ EED: Error estándar de la diferencia entre dos medias. 

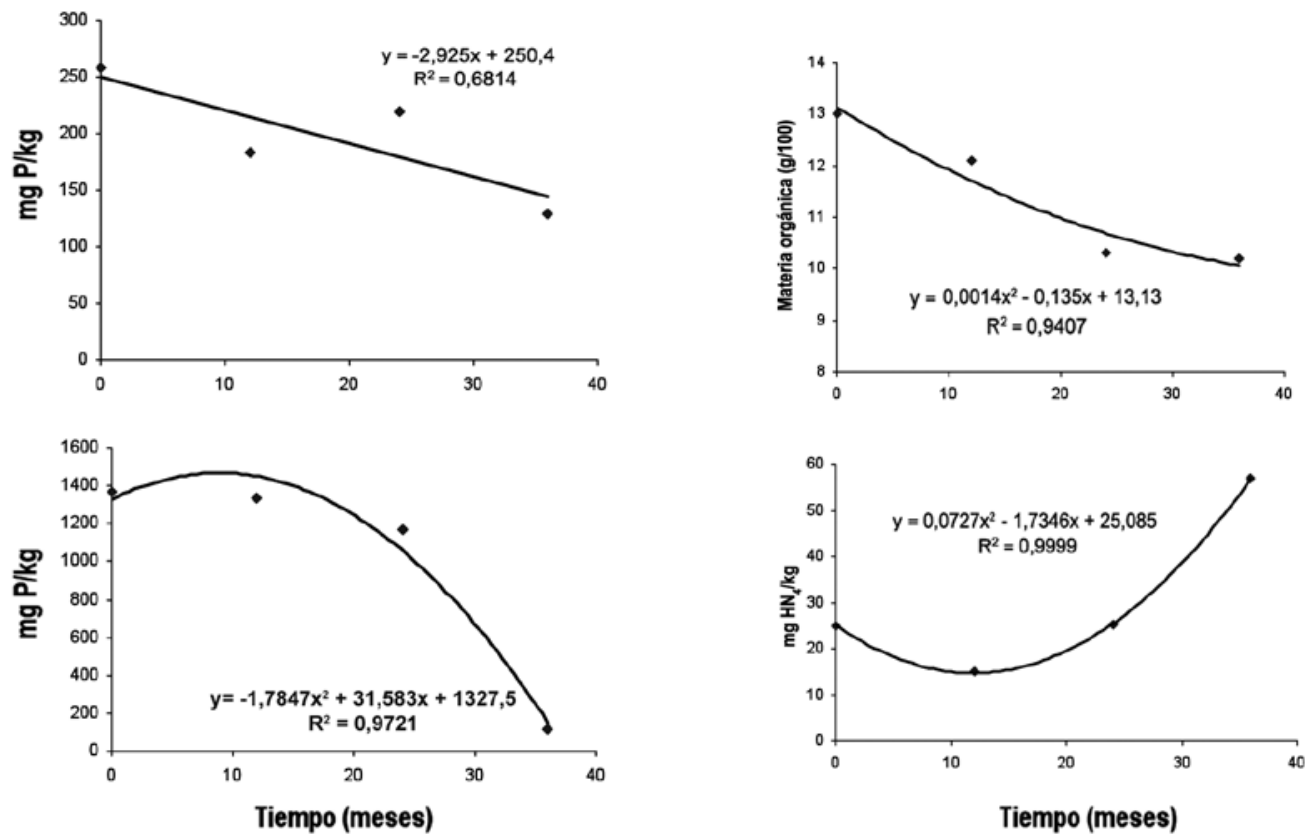

Figura 1. Cambios en las variables del suelo manejado con leguminosas de cobertura asociadas con maíz, durante cuatro ciclos de cultivo consecutivos (1999-2002) en Maní, Yucatán, México.

desarrollo de los cultivos de la milpa en suelos de cultivo continuo (Pool y Hernández 1995).

El marcado incremento de la mineralización de la materia orgánica se atribuye a una relación baja de C/N (Jensen y Castellanos 1994, Armendáriz 1998, Quiroga-Madrigal 2000). En las condiciones particulares de este trabajo no hubo cambios favorables en los nutrimentos y rendimiento de grano de maíz por el cultivo continuo de las leguminosas.

\section{AGRADECIMIENTOS}

Se agradece al Consejo Nacional de Ciencia y Tecnología por el apoyo al proyecto: "Evaluación de leguminosas para intensificar la milpa en Yucatán" clave 980603 y por las becas para la realización de la tesis de Doctorado de José Bernardino Castillo Caamal, de la tesis de Maestría de Manuel Amaya Castro y de la tesis de Lic. En Biología de Rodrigo Rodríguez Carrillo. Un agradecimiento especial a Rosa María Canul Sosa por el apoyo administrativo durante la realización de este trabajo.

\section{LITERATURA CITADA}

Amaya-Castro M; Bautista, F; Castillo, CJ. 2005. Dinámica de la calidad del suelo de la milpa con el uso de leguminosas como cultivo de cobertera. In: Bautista, F; Palacio, G. eds. Caracterización y manejo de los suelos de la Península de Yucatán: Implicaciones agropecuarias, forestales y ambientales. Universidad Autónoma de Campeche, Universidad Autónoma de Yucatán, Instituto Nacional de Ecología. 282 p.

Anderson, J; Ingram, J. 1993. Tropical soil biology and fertility. A handbook of methods. 79-82 CAB International. Wallingford, England. p. 79-82.

Armendáriz-Yañez, IR. 1998. Indigenous fodder legume trees: their influence on soil fertility and animal production on tropical pastures of Yucatan, Mexico. Ph. D. Thesis. University of London, Wye College. $200 \mathrm{p}$.

Ayala-Burgos, AJ; Herrera-Díaz, PE; Castillo-Caamal, JB. 2003. Rumen degradability and chemical composition of the velvet bean (Mucuna spp.) grain and husk. In: M. Eilittä, J. Mureithi, R. Muinga, C. Sandoval y N. Szabo. eds. Increasing Mucuna's Potential as a Food and Feed Crop. Proceedings of an international 
workshop held September 23-26, 2002, in Mombasa, Kenya. Tropical and Subtropical Agroecosystems 1 (2-3):71-76.

Ayala, SA; Basulto, GJA. 2004. Barbechos cultivados para el mejoramiento de la agricultura maicera de roza, tumba y quema en el sur de Yucatán. In: Ojeda NF; Ramírez L. eds. Memorias de I Reunión Estatal de Investigación Agropecuaria y Forestal. Mérida, Yucatán, 15 y 16 de enero del 2004. p. 382-389.

Balam, KM; Bañuelos, RI; García, ME; Gónzalez-Iturbide, AJA; Herrera, CF; Orellana, LR; Vidal, J. 1999. Evaluación Climática. In: Orellana LR ed. Atlas de Procesos Territoriales de Yucatán. Universidad Autónoma de Yucatán. p. 163-182.

Benzing A. 2001. Agricultura orgánica - Fundamentos para la región andina. Ed. Neckar - Verlag, Postfach 1820, 78008 Villingen - Schwenningen, Alemania. 682 p.

Bernabé, SEA. 1998. Leguminosas de cobertera: Mucuna deeringiana (Bort) Merril y Canavalia ensiformis (L.) D. C. asociadas a maíz en San Francisco, La Paz, Santa María Chimalapa, Oaxaca. Tesis de Licenciatura. Universidad Autónoma de Chapingo. México. 124 p.

Binder, U. 1997. Manual de Leguminosas de Nicaragua. Tomo I. PASOLAC Y E.A.G.E. Estelí, Nicaragua. p. 528.

Buckles, D; Barreto, H. J. 1996. Intensificación de sistemas de agricultura tropical mediante leguminosas de cobertura: Un Marco conceptual. CIMMYT/CIAT. Documento 96-06 Es. 13 p.

Buckles, D; Triomphe, B. 1999. Adoption of mucuna in the farming system of northern Honduras. Agroforestry Systems 47:67-91.

Caamal-Maldonado, JA; Jiménez-Osornio, JJ; Torres-Barragán, A; Anaya, AL. 2001. The use allelopathic legume cover and mulch species for weed control in cropping systems. Agronomy Journal 93:27-36.

Carsky, RJ; Tarawali Becker M; Chikoye D; Tian G; Sanginga, N. 1998. Mucuna - herbaceous cover legume with potential for multiple uses. Resource and Crop Management. Research Monograph No. 25. International Institute of Tropical Agriculture. $52 \mathrm{p}$.

Coultas, CL; Post, TJ; Jones, JB Jr; Hsieh, YP. 1996. Use of velvet bean to improve soil fertility and weed control in corn maize production in Northern Belize. Commun. Soil Science and Plant Analysis 27:2171-2196.

Durán, R; Olmsted, I. 1999. Vegetación de la Península de Yucatán. In: Olmsted, I, Gonzáles-Iturbide, JA; Granados, CJ; Olmsted, I; Tun Dzul, F. eds. Atlas de Procesos Territoriales de Yucatán. Universidad Autónoma de Yucatán. p. 183-194.

Drinkwater, L; Cambardella, C; Reeder, J; Rice, C. 1996. Potentially mineralizable nitrogen as an indicator of biologically active soil nitrogen. In: Doran, JW; Jones, AJ. eds. Methods for Assessing Soil Quality. SSSA Special Publication Number 49. Madison Wisconsin, USA. p. 217-229.

Dzib, ALA; Paczka, OR. 2002. Caracterización agronómica del maíz NalXoy. Memorias del XIX Congreso Nacional de Citogenética. Saltillo Coahuila, México. p. 243.

Eilittä, M; Carsky, RJ. 2003. Efforts to improve the potential of Mucuna as a Food and Feed Crop: Background to the Workshop. In: Eilittä, M; Muinga, R; Mureithi, J; Snadoval-Castro; Szabo, N. eds. Increasing mucuna's potential as a food and feed crop. Tropical and Subtropical Agroecosystem 1 (2-3):47-53.

Eilittä, M; Sollenbergër, LE; Littell, RC; Harrington, LW. 2003a. On-farm experiments with maize-mucuna systems in the Los Tuxtlas Region of Veracruz, Mexico. I Mucuna biomass and maize grain yield. Experimental Agriculture 39:5-17.

Eilittä, M; Sollenbergër, LE; Littell, RC; Harrington, LW. 2003b. On-farm experiments with maize-mucuna systems in the Los Tuxtlas Region of Veracruz, Southern Mexico. II Mucuna variety evaluation and subsequent maize grain yield. Experimental Agriculture 39:1927.

Flores, SJ. 2001. Leguminosae (Florística, Etnobotánica y Ecología). Etnoflora yucatanense. Fascículo No. 18. Yucatán, México. 320 p.

Fujii, Y; Shibuya T; Yasuda, T. 1992. Allelopathy of velvetbean: its discrimination and identification of L-DOPA as candidate of allelopathic substances. Japan Agricultural Research Quarterly 25:238-247.

Hernández-X, E; Levy, S; Baltazar, EB. 1995. La rozatumba-quema en Yucatán. In: Hernández-X, E; Bello, E; Levy, S. La milpa en Yucatán: Un sistema de producción agrícola tradicional. Tomo 1 . Colegio de Postgraduados. Estado de México, México. 542 p.

Houba, J; Van Der Lee, J; Novozamsky, I; Walinga, I. 1988. Soil and plants analysis, Part 5, Soil Analysis Procedures. Wageningen University, Wageningen, The Netherlands. 56 p.

Ile, E; Hamadina, MK; Zufa, K; Henfort, J. 1996. Note on effects of a Mucuna pruriens var. Utilis crop on the growth of maize (Zea mays) on an acid ultisol in 
southestern Nigeria. Field Crops Research 48:135140.

Jensen, ES; Castellanos, JZ. 1994. The role of grain legumes in nitrogen cycling of low input sustainable agroecosystems. $15^{\text {th }}$ World Congress of Soil Science. Volumen $5^{\text {a }}$ : Comission IV SYMPOSIA. p. 32-45.

Kessler, DJ. 1990. An agronomica evaluation of jackbean (Canavalia ensiformis) in Yucatan, Mexico. II. Defoliation and time of sowing. Experimental Agriculture 26:23-30.

Lathwell, DJ. 1990. Legume green manure (principles for management based on recent research). Tropsoils Bulletin Number 90-01:5-30.

Lobo-Burle, M; Suhet, AR; Pereira, J; Resk, DVS; José, RR; Cravo, MS; Bowen, W; Bouldin, DR; Lathwell, DJ. 1992. Legume green manures (dry-season survival and the effect on succeeding Maize Crops. Soil Management CRSP Bulletin Number 92-04. 35 p.

Lugo, HJ; García, AMT. 1999. Geomorfología. In: Lugo, HJ. ed. Atlas de procesos territoriales de Yucatán. Universidad Autónoma de Yucatán. p. 155-162.

McDonagh, JE; Hillyer, AE. 2003. Grain legumes in pearl millet systems in Northern Namibia: An assessment of potential nitrogen contributions. Experimental Agriculture 39:349-362.

Martínez, HEI; Morales, L. 1997. Evaluación de leguminosas de coberteras asociadas en el sistema de chahuitera en San Francisco La Paz, Santa María Chimalapa Oaxaca. Tesis de Licenciatura. Universidad Autónoma de Chapingo. México. 107 p.

NAS (National Academy of Sciences). 1979. Tropical legumes: Resources for the future. Washington D. C. 331 p.

Nelson, DW; Sommers, LE. 1982. Total carbon, organic carbon and organic matter. 539-579 p. In: Page, AL; Miller, R H; Keeney, DR. eds. Method of soil analysis, Part 2. American Society of Agronomy, Madison. 961-1010.

Okalebo, R; Gathua, K; Woomer, L. 1993. Laboratory methods of soil analysis: a working manual. KARI, SSSEA, TSBF, UNESCO. Nairobi, Kenya. 88 p.

Parkin, T; Doran, J; Franco-Vizcaíno, E. 1996. Field and laboratory tests of soil respiration. In: Doran, JW et $a l$. ed. Defining soil quality for a sustainable environment. SSSA Special Publication Number 35. Madison Wisconsin, USA. p. 231-245.
Pool NL; Hernández, XE. 1995. Los contenidos de materia orgánica de los suelos en áreas bajo el sistema de roza- tumba-quema: Importancia del muestreo. In Hernández X; Bello BE; Levy TS. eds. La milpa en Yucatán. Un sistema de producción agrícola tradicional. Colegio de Postgraduados Estado de México. Tomo I. p. 109-124.

Pool, NL; León, MNS; González, SCY; Figueroa, FP. 1997. Frijol terciopelo, cultivo de cobertura en la agricultura Chol del Valle de Tulijá, Chiapas, México. Terra 16 (4):359-369.

Quiroga-Madrigal, RR. 2000. Effects of maize (Zea mays L.) cropping systems and tropical legumes on soil chemical and biochemical properties and suppressiveness to soilborne plant pathogens. Ph. D. Thesis, Auburn Alabama. 146 p.

Reyes, CP. 1990. El maíz y su cultivo. A. G. T. Editorial. S. A. México D. F. p. 460.

Romero, GM. 2000. Evaluación de la degradabilidad ruminal de materia seca de rastrojos de maíz criollo en bovinos empleados habitualmente en el trópico. Tesis de licenciatura. Escuela Politécnica Superior de Orihuela. Universidad Miguel Hernández. 66 p.

Sanginga, N; Ibewiro, B; Hougnandan, P; Vanlauwe, B; Okogum, JA. 1996. Evaluation of symbiotic properties contribution of mucuna to maize grown in the derived savanna of West Africa. Plant and Soil. 179:119-129.

Steel, RGD; Torrie, JH. 1988. Bioestadística (principios y procedimientos). McGraw-Hill/Interamericana de México. 2 ed. México D. F. 622 p.

Stern, WR. 1993. Nitrogen fixation and transfer in intercrop system. Field Crops Research 34:335-356.

Terán, S; Rasmussen, CH; May, CO. 1998. Las plantas de la milpa entre los mayas. Fundación Tunben Kin, A.C. Mérida, Yucatán, México. 278 p.

Van Kessel, Ch; Hartley, Ch. 2000. Agricultural management of grain legumes: has it led to an increase in nitrogen fixation?. Field Crops Research. 65: 165-181.

Weisbach, C; Tiessen, H; Jiménez-Osornio, JJ. 2002. Soil fertility during shifting cultivation in the tropical karst soils of Yucatan. Agronomie 22:253-263.

Wortmann, CS; Mcintyre, BD; Kaizzzi, CK. 2000. Annual soil improving legumes: agronomic affectiveness, nutrient uptake, nitrogen fixation and water use. Field Crops Research 68(1):75-83. 